mutation for congenital red cell aplasia or "DBA" in the current study provides important information for genetic counseling of affected families. Clearly, how we name diseases is less important than how we understand them. As diagnostic DNA sequencing becomes increasingly convenient and less expensive, it is more important than ever for practicing physicians to be aware of the clinical consequences of individual gene mutations, the limitations of current knowledge, and the fast-moving new insights that come our way with the advancing technologies of gene discovery.

\section{Acknowledgments}

We thank David Ginsburg for comments on the manuscript. We are grateful to the many patients, families, and referring physicians who have participated in research on DBA and other bone marrow failure disorders. Work on bone marrow failure in our laboratories is supported by funding from the United States Department of Defense (DoD W81XWH-10-1-0974 to M.J. Weiss), the NIH (CA106995 to P.J. Mason and R01 CA105312 to M. Bessler), the Jane Fishman Grinberg Chair in Pediatrics (to M.J. Weiss), and the Buck Family Chair in Hematology (to M. Bessler).
Address correspondence to: Monica Bessler, Buck Family Professor in Hematology, Pediatric and Adult Comprehensive Bone Marrow Failure Center at The Children's Hospital of Philadelphia and The Hospital of the University of Pennsylvania, University of Pennsylvania School of Medicine, 3615 Civic Center Blvd, ARC 302, Philadelphia, Pennsylvania 19104, USA. Phone: 267.426.8782; Fax: 267.426.9892; E-mail: besslerm@email.chop.edu.

1. Diamond L, Blackfan K. Hypoplastic anemia. Am J Dis Child. 1938;56:464-467.

2. Gustavsson P, et al. Diamond-Blackfan anaemia: genetic homogeneity for a gene on chromosome $19 \mathrm{q} 13$ restricted to $1.8 \mathrm{Mb}$. Nat Genet. 1997; 16(4):368-371.

3. Draptchinskaia $\mathrm{N}$, et al. The gene encoding ribosomal protein S19 is mutated in Diamond-Blackfan anaemia. Nat Genet. 1999;21(2):169-175.

4. Narla A, Ebert BL. Ribosomopathies: human disorders of ribosome dysfunction. Blood. 2010; 115(16):3196-3205.

5. Farrar JE, et al. Ribosomal protein gene deletions in Diamond-Blackfan anemia. Blood. 2011; 118(26):6943-6951.

6. Zhang Y, Lu H. Signaling to p53: ribosomal proteins find their way. Cancer Cell. 2009;16(5):369-377.

7. McGowan KA, et al. Ribosomal mutations cause p53-mediated dark skin and pleiotropic effects. Nat Genet. 2008;40(8):963-970.

8. Danilova N, Sakamoto KM, Lin S. Ribosomal protein S19 deficiency in zebrafish leads to developmental abnormalities and defective erythropoiesis through activation of $\mathrm{p} 53$ protein family. Blood.
2008;112(13):5228-5237.

9. Sankaran VG, et al. Exome sequencing identifies GATA1 mutations resulting in Diamond-Blackfan anemia. J Clin Invest. 2012;122(7):2439-2443.

10. Crispino JD. GATA1 in normal and malignant hematopoiesis. Semin Cell Dev Biol. 2005; 16(1):137-147.

11. Ciovacco WA, Raskind WH, Kacena MA. Human phenotypes associated with GATA-1 mutations. Gene. 2008;427(1-2):1-6.

12. Kadri Z, et al. Direct binding of PRb/E2F-2 to GATA-1 regulates maturation and terminal cell division during erythropoiesis. PLoS biology. 2009;7(6):e1000123.

13. Hollanda LM, et al. An inherited mutation leading to production of only the short isoform of GATA-1 is associated with impaired erythropoiesis. Nat Genet. 2006;38(7):807-812.

14. Vlachos A, et al. Diagnosing and treating Diamond Blackfan anaemia: results of an international clinical consensus conference. Br J Haematol. 2008; 142(6):859-876

15. Kee Y, D'Andrea AD. Expanded roles of the Fanconi anemia pathway in preserving genomic stability. Genes Dev. 2010;24(16):1680-1694.

16. Perdahl EB, Naprstek BL, Wallace WC, Lipton JM. Erythroid failure in Diamond-Blackfan anemia is characterized by apoptosis. Blood. 1994; 83(3):645-650.

17. Weiss MJ, Orkin SH. Transcription factor GATA-1 permits survival and maturation of erythroid precursors by preventing apoptosis. Proc Natl Acad Sci. 1995;92(21):9623-9627.

18. Cheng Y, et al. Erythroid GATA1 function revealed by genome-wide analysis of transcription factor occupancy, histone modifications, and mRNA expression. Genome Res. 2009;19(12):2172-2184.

19. Trainor CD, Mas C, Archambault P, Di Lello P, Omichinski JG. GATA-1 associates with and inhibits p53. Blood. 2009;114(1):165-173.

\title{
Lighting the fat furnace without SFRP5
}

\author{
Alexander Rauch and Susanne Mandrup
}

\author{
Department of Biochemistry and Molecular Biology, University of Southern Denmark, Odense, Denmark.
}

\begin{abstract}
WNT signaling plays a central role in the regulation of cellular growth and differentiation. In this issue of the JCI, Mori et al. link WNT signaling to the oxidative capacity of adipocytes during obesity. They show that secreted frizzled-related protein 5 is an extracellular matrix-residing protein that is highly induced during obesity and inhibits oxidative phosphorylation in a tissue-autonomous manner, possibly by sequestering WNT3a. These results implicate local WNT signaling as an attractive target for combating obesity.
\end{abstract}

WNTs are secreted proteins that play important roles in the regulation of many different cellular functions, including growth and development. WNTs signal via frizzled receptors to activate intracellular signaling pathways that lead to the stabilization of $\beta$-catenin (the socalled canonical pathway), or they stimu-

Conflict of interest: The authors have declared that no conflict of interest exists.

Citation for this article: J Clin Invest. 2012; 122(7):2349-2352. doi:10.1172/JCI64196. late various $\beta$-catenin-independent signals, like $\mathrm{Ca}^{2+}$ influx or JNK activation (the noncanonical pathway) (1). Secreted frizzled-related proteins (SFRPs) containing cysteine-rich domains related to those of frizzled receptors negatively regulate WNT signaling by neutralizing WNTs extracellularly (2).

WNT signaling has previously been reported to play an important role in adipocyte differentiation. The activation of $\beta$-catenin by WNTs, including WNT6, WNT10a, and WNT10b, blocks adipo- cyte differentiation $(3,4)$. In contrast, the effects of the putative noncanonical ligands WNT4 and WNT5a on adipocyte differentiation remain the subject of controversy. It has been shown that knockdown of WNT4 or WNT5a diminishes adipocyte differentiation of 3T3-L1 preadipocytes (5); however, suppressive actions of WNT5a treatment have also been reported (6).

In this issue of the JCI, Mori, MacDougald, and colleagues report an unexpected role of SFRP5 on the oxidative capacity of adipocytes in vivo and ex vivo (7). The authors confirmed previous findings that Sfrp5 mRNA expression is restricted to adipocytes within white adipose tissue $(8,9)$ and showed that Sfrp5 expression was induced during late stages of adipocyte differentiation. Furthermore, they found that Sfrp5 mRNA expression was upregulated 

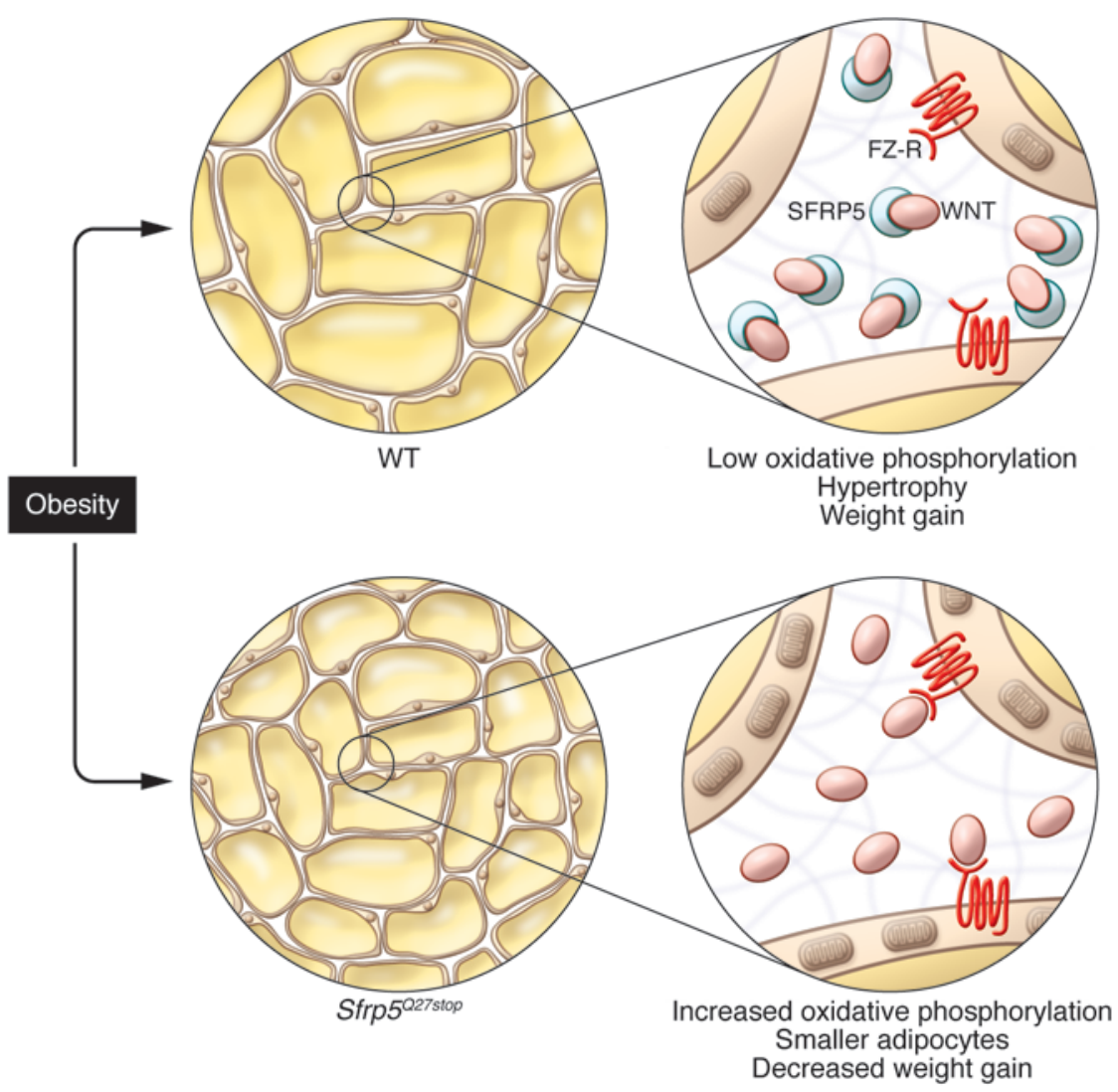

in several mouse models of obesity, with a strong correlation between adipocyte size and Sfrp5 transcript levels.

\section{SFRP5 blocks the fat furnace}

To further investigate the role of SFRP5 in adipose biology, the authors used a mouse model with an $N$-ethyl- $N$-nitrosourea-generated point mutation in the Sfrp5 locus that causes a premature stop codon in the first exon (Sfrp5 227stop mice). These SFRP5deficient mice were indistinguishable from their WT littermates, and mesenchymal stem cells isolated from Sfrp5027stop and WT mice displayed similar adipogenic differentiation (7). However, when challenged with a high-fat diet (HFD), most Sfrp $5^{\text {Q27stop mice }}$ gained less weight and were more insulin sensitive than WT littermates. The resistance to obesity was based on decreased appearance of large hypertrophic adipocytes in response to the HFD. This effect is at least primarily tissue autonomous, since it was recapitulated in adipose tissue transplants independent of the genetic background of the host. In support of primarily paracrine/autocrine effects in the adipose tissue, SFRP5 was only detected in the extracellular matrix, not in the supernatant, of adipocyte cultures.
Intriguingly, Mori et al. found that the

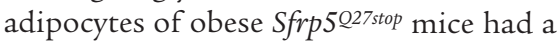
higher oxidative capacity, due to increased number as well as increased oxidative capacity of mitochondria (7). Importantly, this effect must be cell autonomous, since adipocytes differentiated in vitro from mesenchymal precursors of Sfrp5 ${ }^{\text {Q27stop }}$ mice maintained their oxidative phenotype with respect to gene expression and oxygen consumption. Thus, the loss of SFRP5 leads to an increase in mitochondrial oxidative capacity in adipocytes, which in turn diminishes the accumulation of lipids while on HFD (Figure 1).

High expression levels of Sfrp5 have previously been reported as the best a priori predictor for a large increase in body weight in genetically identical mice (10). In line with this, the present study convincingly demonstrated that in many models of obesity, including $d b / d b, o b / o b$, ovariectomized, and HFD-fed mice, increased Sfrp5 transcript levels in adipose tissues correlated with adipocyte size (7). Similar upregulation of Sfrp5 protein was documented after 12 weeks of HFD by Ouchi, Walsh, and colleagues

\section{Sfrp5 expression in obesity}

\section{Figure 1}

SFRP5 is an adipocyte-derived, matrix-residing protein that sequesters WNTs to reduce WNT signaling. Endogenous WNTs stimulate the oxidative capacity of adipocytes by increasing mitochondrial number and activity. During obesity, SFRP5 levels rise, thereby suppressing the oxidative metabolism of adipocytes, which subsequently leads to adipocyte hypertrophy. In the absence of SFRP5 (Sfrp5Q27stop), adipocytes keep their oxidative potential, which leads to an overall shift to smaller adipocytes and thus limits weight gain. FZ-R, frizzled receptor.

(8). Notably, however, suppression of SFRP5 expression was observed after 24 weeks of HFD (8).

Mori et al. showed that Sfrp5 expression was induced during adipocyte differentiation (7). In addition, Sfrp5 transcript levels in adipose tissue were shown to correlate with adipocyte size (7). Consistent with this, Sfrp5 expression has been previously shown to be induced by refeeding (11).

It is unclear what transcription factors account for the induction during differentiation, and chromatin occupancy analyses of adipogenic transcription factors have not demonstrated binding close to the Sfrp5 locus $(12,13)$. Similarly, the mechanisms inducing expression of the gene during obesity and in response to refeeding are unknown. It is possible that insulinactivated factors such as sterol-regulatory element binding proteins play a role; however, proinflammatory signals, secondary to the increase in adipocyte size, could also be involved during obesity.

\section{Function of SFRP5 in obesity}

The results of the present study differ from those of the Walsh group in the reported effects of genetic ablation of Sfrp5, despite a similar genetic background and a com- 
parable dietary regime. In contrast to the antiobesity phenotype of the Sfrp5Q27stop mice, the Sfrp5 $5^{-/-}$mice used by the Walsh group displayed an increase in adiposity, adipocyte size, inflammatory response, and insulin resistance after exposure to HFD (8). Of note, this metabolic phenotype of obese Sfrp5 $5^{-/-}$mice was assessed after 12 weeks of HFD, at which time Sfrp5 expression was elevated in WT littermate controls. The reason for the difference between the phenotypes is unknown, since neither of the two targeting strategies is predicted to have off-target effects on other genes. The Sfrp5Q27stop mice were generated by chemical mutagenesis (14) and backcrossed to C57BL/ 6 for approximately 20 generations; whereas the Sfrp $5^{-/-}$mice were generated by replacing exon 1 with a $P G K$ neobpAloxA cassette (15).

Moreover, the two studies also differ in their suggested mechanisms of action. Mori et al. demonstrated that ectopically expressed SFRP5 was mainly associated with the extracellular matrix in cell cultures (7). Furthermore, adipose tissue transplants deficient in SFRP5 protein could not be rescued by high levels of host SFRP5 from $d b / d b$ mice (7). This is consistent with the notion that WNTs/SFRPs primarily act in an autocrine and paracrine manner. Conversely, the Walsh group reported that ectopically expressed SFRP5 is secreted to the medium and serum, where it acts systemically; moreover, adenovirally expressed SFRP5 protein could rescue the phenotype of Sfrp $5^{-1-}$ mice (8). Thus, it appears that the primary direct mechanism of action in the adipose tissue is autocrine/paracrine signaling. However, systemic administration of SFRP5 may also affect adipose tissues, possibly by indirect mechanisms via other tissues. Mori et al. suggest that SFRP5 decreases mitochondrial oxidative capacity in adipocytes and that this defect is amplified during obesity as a result of increased SFRP5 levels (7).

\section{Fishing for the escaping WNT}

An important question as to which WNT ligand(s) mediate the effect of SFRP5 deficiency remains to be resolved. Mori et al. report that treatment with purified recombinant WNT3a, a putative canonical WNT, recapitulated the cell-autonomous effects of SFRP5 deficiency on adipocytes, including increased expression of genes involved in oxidative phosphorylation (7). Notably, however, the WNT3a-treated cells showed an increase in basal oxidative metabolism rather than their maximal capacity, which suggests that WNT3a administration leads to induction of slightly different signals than does SFRP5 deficiency. In contrast, the noncanonical WNT5a was reported to account for the effects seen by the Walsh group (8). Both WNTs have been shown to affect mesenchymal stem cell fate by suppressing adipocyte differentiation. WNT3a has been reported to induce COUP-TFII, which represses Pparg expression (16), whereas WNT5a signaling directs corepressor complexes to PPAR $\gamma$ target sites, thereby blunting PPAR $\gamma$ transactivation (6). It is unclear how this repression of PPAR $\gamma$ activity would promote obesity in the study by the Walsh group (8).

Walsh and colleagues reported that SFRP5 counteracts WNT5a-mediated noncanonical activation of JNK, the activity of which is increased by obesity. Consistent with this, Sfrp $5^{-/-}$mice lose their phenotype on a Jnk1-/- background (8). However, the interpretation is complicated, since HFDfed $J n k 1^{-/-}$mice are resistant to developing obesity (17), meaning that these mice would mimic the lean situation, in which ablation of SFRP5 does not affect the metabolic phenotype. To strengthen the argument for a SFRP5-JNK axis, it would be important to determine the cell types involved, as JNK activity in different cell types has been shown to contribute to obesity and insulin resistance (17). Mori et al. demonstrated that SFRP5 loss of function did not alter JNK activity in obese mice, which indicates that SFRP5 is not a major regulator of JNK. Conversely, the authors showed that WNT3a signaling could mimic the effects of loss of SFRP5 (7). Neither the Walsh group nor the MacDougald group has yet demonstrated direct binding of SFRP5 to WNT3a or WNT5a. However, SFRP5 was previously shown to bind WNT5a using overexpression of tagged proteins (18), and SFRP5 is likely to bind WNT3a based on in silico analyses using a human protein-protein interaction prediction database, PIP $(19,20)$. It has also been shown that WNT3a and WNT5a bind and trigger phosphorylation events at different subsets of coreceptors, LRP5/6 and ROR1/2, respectively (21). Thus, understanding coreceptor availability and activation would provide some insight into the putative WNT signaling pathways affected by SFRP5.

In summary, the link between global Sfrp5 deletion and the action of the putative WNT ligands on adipose tissue is weak.
For example, it has not been shown that elevation of WNT3a or WNT5a levels in the adipose tissue causes a phenotype similar to that of SFRP5 loss. It is also possible that the action of SFRP5 on adipocytes is independent of WNT ligands, as has been reported for other SFRPs (22).

\section{Lingering questions}

In addition to resolving the conflicting data obtained from different Sfrp5-deficient mouse models, a future challenge will be to obtain insight into the regulation of Sfrp5 expression. It will be particularly interesting to uncover the mechanisms underlying the increased expression in response to obesity and refeeding, conditions that are both associated with high insulin levels. Furthermore, it will be important to determine which WNT signaling pathways are affected by SFRP5 deficiency in adipose tissue and to uncover the mechanisms by which SFRP5 and WNT signaling modulates nuclear and mitochondrial gene expression involved in oxidative metabolism.

\section{Acknowledgments}

A. Rauch is supported by an EMBO longterm fellowship.

Address correspondence to: Susanne Mandrup, Campusvej 55, DK-5230 Odense M, Denmark. Phone: 45.6550.2340; Fax: 45.6550. 2467; E-mail: s.mandrup@bmb.sdu.dk.

1. Kikuchi A, Yamamoto H, Sato A, Matsumoto S. Wnt5a: its signalling, functions and implication in diseases. Acta Physiol (Oxf). 2012;204(1):17-33.

2. Rattner A, et al. A family of secreted proteins contains homology to the cysteine-rich ligand-binding domain of frizzled receptors. Proc Natl Acad SciUS A. 1997;94(7):2859-2863.

3. Ross SE, et al. Inhibition of adipogenesis by Wnt signaling. Science. 2000;289(5481):950-953.

4. Cawthorn WP, et al. Wnt6, Wnt10a and Wnt10b inhibit adipogenesis and stimulate osteoblastogenesis through a beta-catenin-dependent mechanism. Bone. 2012;50(2):477-489.

5. Nishizuka M, Koyanagi A, Osada S, Imagawa M. Wnt4 and Wnt5a promote adipocyte differentiation. FEBS Lett. 2008;582(21-22):3201-3205.

6. Takada I, et al. A histone lysine methyltransferase activated by non-canonical Wnt signalling suppresses PPAR-gamma transactivation. Nat Cell Biol. 2007;9(11):1273-1285.

7. Mori $\mathrm{H}$, et al. Secreted frizzled-related protein 5 suppresses adipocyte mitochondrial metabolism through WNT inhibition. J Clin Invest. 2012; 122(7):2405-2416

8. Ouchi N, et al. Sfrp5 is an anti-inflammatory adipokine that modulates metabolic dysfunction in obesity. Science. 2010;329(5990):454-457.

9. Schulte DM, et al. Pro-inflammatory wnt5a and anti-inflammatory sFRP5 are differentially regulated by nutritional factors in obese human subjects. PLoS One. 2012;7(2):e32437.

10. Koza RA, et al. Changes in gene expression fore- 
shadow diet-induced obesity in genetically identical mice. PLoS Genet. 2006;2(5):e81.

11. Lagathu C, et al. Dact1, a nutritionally regulated preadipocyte gene, controls adipogenesis by coordinating the Wnt/beta-catenin signaling network. Diabetes. 2009;58(3):609-619.

12. Nielsen R, et al. Genome-wide profiling of PPARgamma:RXR and RNA polymerase II occupancy reveals temporal activation of distinct metabolic pathways and changes in RXR dimer composition during adipogenesis. Genes Dev. 2008; 22(21):2953-2967.

13. Siersbaek R, et al. Extensive chromatin remodelling and establishment of transcription factor 'hotspots' during early adipogenesis. EMBOJ. 2011; 30(8):1459-1472

14. Quwailid MM, et al. A gene-driven ENU-based approach to generating an allelic series in any gene. Mamm Genome. 2004;15(8):585-591.

15. Satoh W, Gotoh T, Tsunematsu Y, Aizawa S, Shimono A. Sfrp1 and Sfrp2 regulate anteroposterior axis elongation and somite segmentation during mouse embryogenesis. Development. 2006; 133(6):989-999.

16. Okamura M, et al. COUP-TFII acts downstream of Wnt/beta-catenin signal to silence PPARgamma gene expression and repress adipogenesis. Proc Natl Acad Sci U S A. 2009;106(14):5819-5824.

17. Solinas G, et al. JNK1 in hematopoietically derived cells contributes to diet-induced inflammation and insulin resistance without affecting obesity. Cell Metab. 2007;6(5):386-397.

18. Li Y, Rankin SA, Sinner D, Kenny AP, Krieg PA, Zorn AM. Sfrp5 coordinates foregut specification and morphogenesis by antagonizing both canonical and noncanonical Wnt11 signaling. Genes Dev. 2008;22(21):3050-3063.

19. Scott MS, Barton GJ. Probabilistic prediction and ranking of human protein-protein interactions. BMC Bioinformatics. 2007;8:239.

20. McDowall MD, Scott MS, Barton GJ. PIPs: human protein-protein interaction prediction database. Nucleic Acids Res. 2009;37(database issue):D651-D656.

21. Grumolato L, et al. Canonical and noncanonical Whts use a common mechanism to activate completely unrelated coreceptors. Genes Dev. 2010; 24(22):2517-2530.

22. Bovolenta P, Esteve P, Ruiz JM, Cisneros E, LopezRios J. Beyond Wnt inhibition: new functions of secreted Frizzled-related proteins in development and disease. J Cell Sci. 2008;121(pt 6):737-746.

\title{
Oxidative stress and intracellular infections: more iron to the fire
}

\author{
Norma W. Andrews \\ Department of Cell Biology and Molecular Genetics, University of Maryland, College Park, College Park, Maryland, USA.
}

\begin{abstract}
The immune system's battle against pathogens includes the "respiratory burst," a rapid release of ROS from leukocytes, thought to play a role in destroying the invading species. In this issue of the JCI, Paiva et al. demonstrate that oxidative stress actually enhances infection with the protozoan Trypanosoma cruzi, by a mechanism that may involve facilitating parasite access to iron. Their findings suggest a novel direction for the development of drugs against intracellular parasites.
\end{abstract}

The immune system of higher vertebrates is capable of rapidly recognizing pathogens, mounting an immediate innate response. The innate response is followed by adaptive immunity, the slower-developing process that is responsible for preventing reinfection by the same organism. An important component of the rapid innate immune response is production of ROS, highly reactive and toxic molecules produced by phagocytes and other cell types. ROS have been traditionally viewed as a "necessary evil" in the battle against pathogens, and their production is coupled to antioxidant responses important for mitigating oxidative damage in cells and tissues. However, this is clearly not the whole story. In this issue of the JCI, Paiva et al. (1) demonstrate that antioxidant responses actually suppress infections with Trypanosoma cruzi, a protozoan parasite that seems to thrive in an oxidative environment. Earlier studies with viruses and bacterial

Conflict of interest: The author has declared that no conflict of interest exists.

Citation for this article: J Clin Invest. 2012; 122(7):2352-2354. doi:10.1172/JCI64239. pathogens suggested a similar scenario, but those observations were mostly attributed to a role of antioxidant pathways in innate immunity. Challenges to established dogma are usually slow to emerge, but this detailed T. cruzi study raises the intriguing possibility that the deleterious effects of antioxidants on intracellular pathogens may occur by a mechanism fundamentally different from classical innate or adaptive immune responses.

\section{The role of oxidative stress}

This insight came from experiments wherein mice and mouse-derived macrophages were exposed to CoPP, an activator of the transcription factor NRF2 that orchestrates antioxidant responses. NRF2 drives expression of heme oxygenase-1 (HO-1), an enzyme that shifts the cellular redox balance by degrading prooxidant heme (2). Surprisingly, activation of NRF2 and increased expression of HO-1 markedly reduced parasite burden in infected animals and in isolated macrophages. The process was independent of $\mathrm{T}$ lymphocyte-mediated immunity and did not seem to involve apoptotic clearance of infected cells or effectors known to be active against $T$. cruzi, such as NO, TNF, or type I IFN (1). Remarkably, NRF2 activation was still able to reduce the very high T. cruzi load that is observed in iNOSdeficient mice. NRF2 activation had the expected effect of inhibiting ROS generation in infected macrophages, but this did not affect the number of intracellular parasites found shortly after infection (1). This observation is consistent with the presence in T. cruzi and other trypanosomatid parasites of a unique and highly effective antioxidant machinery, the trypanothione-thiol system (3).

The unexpected role of high oxidative stress in promoting T. cruzi infection may have important implications for the pathology of Chagas disease, the chronic debilitating illness that affects millions of people infected by this parasite in Latin America. Although infective trypomastigote stages can invade practically every nucleated cell type, in vivo the parasites are frequently found replicating in skeletal and cardiac muscle (4). The most serious manifestation of chronic Chagas disease, cardiomyopathy, is responsible for significant mortality in infected patients and has been directly attributed to the persistence of T. cruzi within cardiomyocytes $(5,6)$. Interestingly, there is evidence that marked and sustained oxidative stress is established in cardiomyocytes following T. cruzi infection, due to parasite-induced disturbances in mitochondrial membrane potential and electron 